Z Herz- Thorax- Gefäßchir 2021 · 35:253-254 https://doi.org/10.1007/s00398-021-00460-z Angenommen: 6. September 2021

(c) Springer Medizin Verlag $\mathrm{GmbH}$, ein Teil von Springer Nature 2021

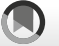

\section{Wie aus Leitlinien Leidlinien werden!}

\author{
Jochen Cremer \\ Klinik für Herz- und Gefäßchirurgie, Universitätsklinikum Schleswig-Holstein, Campus Kiel, Kiel, \\ Deutschland
}
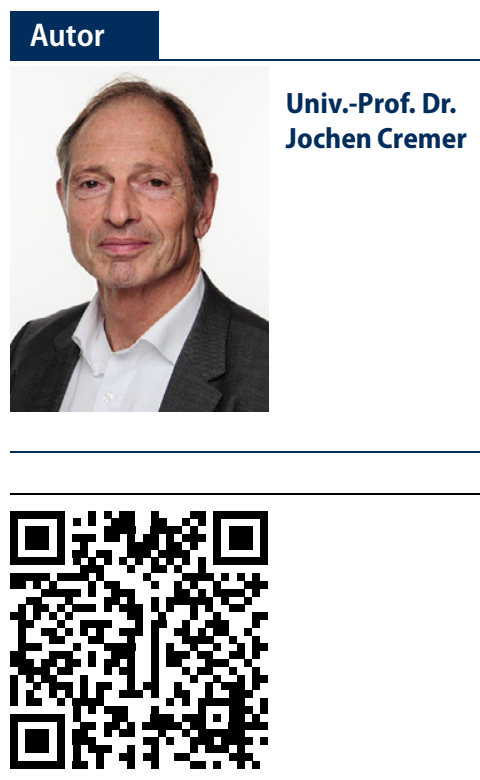

QR-Code scannen \&Beitrag online lesen
Liebe Leserinnen und Leser,

worauf ich hinaus will: Mit Veröffentlichung der neuen „2021 ESC/EACTS Guidelines for the management of valvular heart disease" ist ganz offensichtlich ein doppeltes gravierendes Dilemma entstanden, das dann mindestens 2 grundsätzliche Fragen aufwirft: Was wird als wissenschaftliche Evidenz angesehen, und wie wird in den verantwortlichen Gremien wissenschaftlicher Fachgesellschaften damit umgegangen? Das eigentliche Dilemma liegt dabei aber nicht in den neuen ESC/EACTS Guidelines, die nach meiner Meinung sehr korrekt und tatsächlich wissenschaftlich evident sind, sondern in dem, was zwischenzeitlich, also nach Erscheinen der „2017 ESC/EACTS Guidelines for the management of valvular heart disease" für die USA und für Deutschland, erschienen ist.

Also im Detail: Bei „behandlungsbedürftiger" Aortenstenose empfehlen die ESC/EACTS für Patienten jünger als 75 Jahre mit niedrigem Risiko für SAVR (Surgical Aortic Valve Replacement) oder für Patienten, die nicht für eine transfemorale TAVI (Transcatheter Aortic Valve Implantation) geeignet und operabel sind, die chirurgische Aortenklappenimplantation (SAVR). Für Patienten über ( $\geq$ ) 75 Jahre oder mit hohem Risiko (bzw. ungeeignet) für eine SAVR und geeignet für eine transfemorale TAVI, wird die TAVI empfohlen. Alle anderen Patienten sollen eine SAVR oder eine TAVI erhalten.

So weit so gut.

Sieht man sich jetzt das „Guideline Update on Indications for Transcatheter Aortic Valve Implantation Based on the 2020 American College of Cardiology/American Heart Association Guidelines for Management of Valvular Heart Disease" an, das zudem zeitlich früher (im Dezember 2020) veröffentlicht wurde, so findet sich hier im Evidenzlevel A eine deutlich abweichende Empfehlungslage mit Altersdenominatoren von 65 und 80 Jahren und kombinierten Lebenserwartungen von 12 Monaten, 10 Jahren und 20 Jahren, also offensichtlich ganz ohne formale Diskussion der Operationsrisiken.

Demzufolge wird SAVR empfohlen für Patienten jünger als 65 Jahre mit einer Lebenserwartung von über 20 Jahren (whoever that is!).

Für Patienten zwischen 65 und 80 Jahren wird SAVR oder TAVI empfohlen, und für Patienten älter als 80 Jahre mit einer Lebenserwartung unter 10 Jahren wird die TAVI empfohlen. Es mag sowieso schwierig sein, diese Gruppe zu kalkulieren, und darüber hinaus wird dann keine Empfehlung für Patienten über 80 Jahre und mit einer Lebenserwartung über 10 Jahren (in logischer Konsequenz müsste es ja auch diese Patienten geben) mehr ausgesprochen, ganz offensichtlich, weil schon genug TAVI-Terrain erobert wurde. In der entsprechenden Publikation heißt es dann unter EVIDENCE BASE .... "Large quantities of data are now available from RCTs (randomized controlled trial), registries and observational studies comparing TAVI with medical therapy as well as SAVR in patients older than 65 years."

In der Literatur dazu finden sich dann zwar dieselben Studien und Publikationen wie in den ESC/EACTS Guidelines, übrigens aber nur Partner I, Partner II und Corevalve US sowie die Metaanalyse von Siontis aus dem EHJ 2019 und keine der angesprochenen "registries" und "observational studies“. Der Punkt "durability“ im Zusammenhang mit der "life expectancy" 
bis 20 Jahre wird genau so wenig adressiert.

Man wundert sich dann schon, wie dieses Update zustande kommt, wo das mittlere Alter in Partner I, Partner Il und Corevalve US doch zwischen 81,5 und 84,5 Jahren liegt und sich der Nachbeobachtungszeitraum auf nur 5 Jahre erstreckt.

Es drängt sich also der substanzielle Eindruck auf, dass diese Updates aus dem FF entwickelt wurde, also wohl eher auf der Basis von "fake facts". Zu allem Überfluss gibt es dann noch davon, also von den ESC/EACTS Guidelines 2017 und 2021 und dem Guideline Update ACC/AHA abweichende Positionen im DGK-DGTHG-Konsensuspapier von 2020 wohl nur für die deutsche Szene hinsichtlich der Indikationsstellung für SAVR und TAVI mit relevanten Altersgrenzen - man wundere sich bei dann wiederum 70 und 75 Jahren und einer Bewertung der Lebensperspektive scheint ja was anderes als Lebenserwartung zu sein. Im Klartext wird SAVR primär für Niedrigrisikopatienten unter $(<) 70$ Jahren empfohlen, während bei 70- bis 75Jährigen das Feld offen für TAVI oder AKE ist.

Es stellt sich dann schon die Frage, ob jetzt auf der Basis der 2021 ESC/EACTS Guidelines das DGK-DGTHG-Konsensuspapier wieder überarbeitet werden muss, bei ganz offensichtlichem Widerspruch bezüglich der Diskussionskorridore.

Aber dennoch zum Trost, geteiltes Leid ist halbes Leid.

Oder vielmehr geteiltes Leit ist doppeltes Leid.

Ihr

Jochen Cremer

\section{Korrespondenzadresse}

\section{Univ.-Prof. Dr. Jochen Cremer}

Klinik für Herz- und Gefäßchirurgie,

Universitätsklinikum Schleswig-Holstein,

Campus Kiel

Arnold-Heller-Straße 3, Haus C, 24105 Kiel, Deutschland

jochen.cremer@uksh.de

Interessenkonflikt. J. Cremer gibt an, dass kein Interessenkonflikt besteht.

\section{Weiterführende Literatur}

1. The Task Force for the Management of Valvular Heart Disease of the European Society of Cardiology (ESC), the European Association for Cardio-Thoracic Surgery (EACTS), Baumgartner H, Falk V, Bax JJ, De Bonis M, Hamm C, Holm PJ, lung B, Lancellotti P, Lansac E, Rodriguez Munoz D, Rosenhek R, Sjögren J, Tornos Mas P, Vahanian A, Walther T, Wendler O, Windecker S, Zamorano JL (2017) 2017 ESC/EACTS Guidelines for the management of valvular heart disease, European Journal of Cardio-Thoracic Surgery 52 (2017) 616-664

2. Developed by the Task Force for the management of valvular heart disease of the European Society of Cardiology (ESC) and the European Association for Cardio-Thoracic Surgery (EACTS); Authors/Task Force Members: Alec Vahanian (ESC Chairperson) (France), Friedhelm Beyersdorf (EACTS Chairperson) (Germany), Fabien Praz (ESC Task Force Coordinator) (Switzerland), Milan Milojevic (EACTS Task Force Coordinator) (Serbia), Stephan Baldus, Johann Bauersachs, Davide Capodanno, Lenard Conradi, Michele De Bonis, Ruggero De Paulis, Victoria Delgado, Nick Freemantle, Martine Gilard, Kristina H. Haugaa, Anders Jeppsson, Peter Juni, Luc Pierard, Bernard D. Prendergast, J. Rafael Sadaba, Christophe Tribouilloy, Wojtek Wojakowski,2021 ESC/EACTS Guidelines for the management of valvular heart disease.European Journal of Cardio-Thoracic Surgery 00 (2021) 1-74

3. Sundt TM, Jneid H (2021) Guideline Update on Indications for Transcatheter Aortic Valve Implantation Based on the 2020 American College of Cardiology/American Heart Association Guidelines for Management of Valvular Heart Disease. jama cardiol. https://doi.org/10.1001/ jamacardio.2021.2534

4. Kuck K-H, Bleiziffer $S$, Eggebrecht $H$, Ensminger $S$, Frerker C, Möllmann H, Nef H, Thiele H, Treede H, Wimmer-Greinecker GTW (2020) Konsensuspapier der Deutschen Gesellschaft für Kardiologie (DGK) und der Deutschen Gesellschaft für Thorax-, Herz- und Gefäßchirurgie (DGTHG) zur kathetergestützten Aortenklappenimplantation (TAVI). Kardiologe 2020(14):182-204

5. Siontis GCM, Overtchouk P, Cahill TJ et al (2019) Transcatheter aortic valve implantation vs. surgical aortic valve replacement for treatment of symptomatic severe aortic stenosis: an updated meta-analysis. Eur Heart J 40(38):3143-3153. https://doi.org/10.1093/eurheartj/ehz275

6. Mack MJ, Leon MB, Smith CR et al (2015) 5-year outcomes of transcatheter aortic valve replacement or surgical aortic valve replacement for high surgical risk patients with aortic stenosis (PARTNER 1): a randomised controlled trial. Lancet 385:2477-2484

7. Makkar RR, Thourani VH, Mack MJ et al (2020) Fiveyear outcomes of transcatheter or surgical aorticvalve replacement. J Med 382:799-809

8. Gleason TG, Reardon MJ, Popma JJ et al (2018) SelfExpanding Transcatheter Aortic Valve Replacement or Surgical Valve Replacement in High-Risk Patients. J Am Coll Cardiol 72(22):2687-2696
Auch online Zugang zu allen Beiträgen Ihres Zeitschriftenabonnements

Wussten Sie, dass Sie als Abonnent*in dieser Zeitschrift automatisch Online-Zugriffsrechte auf das gesamte Beitragsarchiv und die CMEKurse haben?

Der Zugang zu Ihrer Online-Bibliothek und den CME-Kursen ist im Abonnement Ihrer Zeitschrift inbegriffen. Für den Zugang müssen Sie sich lediglich einmal über www.springermedizin.de/register registrieren.

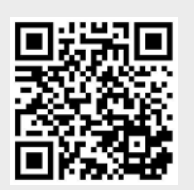

Über diesen QR-Code schnell und einfach registrieren

Bitte nutzen Sie für die Registrierung Vorund Nachname und Lieferadresse wie beim Abonnement der Zeitschrift (s. Adressaufkleber auf Ihrem Heft). So kann im System die Zugehörigkeit zu Ihrer Zeitschrift sichergestellt werden.

Aufgrund des Heilmittelwerbegesetzes dürfen die Inhalte der Website nur medizinischen Fachkreisen zur Verfügung gestellt werden. Bei der Anmeldung bitten wir Sie deshalb, einen Berufsnachweis vorzulegen. Bei einer Mitgliedschaft in der deutschen Ärztekammer reicht die Angabe der einheitliche Fortbildungsnummer (EFN). Alternativ schicken Sie eine Bestätigung des Arbeitgebers, Studiennachweis mit Angabe des Studiengangs ganz unkompliziert an kundenservice@springermedizin.de.

Mit Benutzername und Passwort haben Sie außerdem Zugang zu den freien Inhalten auf den Seiten von: www.springermedizin.de www.aerztezeitung.de

Sollten Fragen oder Probleme auftauchen, wenden Sie sich einfach an Ihren Kundenservice:

kundenservice@springermedizin.de 